\title{
Reconstruction and classification of tau lepton decays with ILD
}

\author{
T. H. Tran ${ }^{\text {a }}$, V. Balagura, V. Boudry, J.-C. Brient, H. Videau \\ Laboratoire Leprince-Ringuet, École polytechnique, CNRS/IN2P3, Université Paris-Saclay, 91128 Palaiseau, France
}

Received: 21 October 2015 / Accepted: 10 August 2016 / Published online: 20 August 2016

(C) The Author(s) 2016. This article is published with open access at Springerlink.com

\begin{abstract}
Tau lepton decays with up to two $\pi^{0} \mathrm{~s}$ in the final state $-\tau^{+} \rightarrow \pi^{+} \bar{\nu}_{\tau}, \rho^{+}\left(\pi^{+} \pi^{0}\right) \bar{\nu}_{\tau}$, and $\mathrm{a}_{1}^{+}\left(\pi^{+} \pi^{0} \pi^{0}\right) \bar{\nu}_{\tau}-$ are used to study the performance of the barrel region of the silicon-tungsten electromagnetic calorimeter ( $\mathrm{Si}-\mathrm{W}$ ECAL) of the International Large Detector (ILD) at the future $\mathrm{e}^{+} \mathrm{e}^{-}$ International Linear Collider. Correct reconstruction of the tau decay mode is crucial for constraining the spin state of tau lepton and measuring the Higgs boson CP state in $\mathrm{H} \rightarrow \tau^{+} \tau^{-}$decays. We find that about $95 \%$ of $\pi^{+} \bar{\nu}_{\tau}$, and $90 \%$ of $\rho^{+} \bar{\nu}_{\tau}$ and $\mathrm{a}_{1}^{+} \bar{\nu}_{\tau}$ decays produced by the $\mathrm{e}^{+} \mathrm{e}^{-} \rightarrow$ $\mathrm{Z}^{0^{*}} \rightarrow \tau^{+} \tau^{-}$process at an $e^{ \pm}$beam energy of $125 \mathrm{GeV}$ are correctly reconstructed. In a smaller ILD detector, with the inner $\mathrm{Si}-\mathrm{W}$ ECAL radius reduced by about $20 \%$, these efficiencies are reduced by at most $2 \%$. The $\pi^{0}$ mass resolution remains below $10 \%$. Since failures in tau lepton reconstruction are mainly due to photons, an increase of the ILD magnetic field from 3.5 to $4 \mathrm{~T}$ does not bring any significant improvement.
\end{abstract}

\section{Introduction}

Tau lepton decays have been used at LEP [1] and many other experiments for precise tests of the Standard Model. The spin state of the tau lepton can be inferred from its decay mode and the momenta of all reconstructed decay products [2]. This will be used at future lepton colliders, for example, to measure the $\mathrm{CP}$ (the product of charge conjugation and parity symmetries) state of the Higgs boson decaying into a tau pair, $\mathrm{H}^{0} \rightarrow \tau^{+} \tau^{-}$. The tau decay products are usually more collimated than QCD jets of similar energy, and the separation of photon clusters in the electromagnetic calorimeter (ECAL) is more difficult. Incorrect determination of the number of photons in the tau final state results in the wrong reconstruction of the tau decay mode and degrades the spin state measurement.

\footnotetext{
a e-mail: trong.hieu.tran@1lr.in2p3.fr
}

The reconstruction of the tau decay photons is therefore particularly suited as a benchmark of the ECAL performance.

In this paper we study the reconstruction and classification of $\tau^{+}$decay modes ${ }^{1}$ in the international large detector (ILD), a detector concept for the $\mathrm{e}^{+} \mathrm{e}^{-}$international linear collider (ILC). We concentrate on the reconstruction of three main tau decays with one charged pion and up to two $\pi^{0} \mathrm{~s}$ : $\tau^{+} \rightarrow \pi^{+} \bar{v}_{\tau}, \rho^{+} \bar{v}_{\tau}$, and $\mathrm{a}_{1}^{+} \bar{v}_{\tau}$, followed by $\rho^{+} \rightarrow \pi^{+} \pi^{0}$, $\mathrm{a}_{1}^{+} \rightarrow \pi^{+} \pi^{0} \pi^{0}$, and $\pi^{0} \rightarrow \gamma \gamma$. For simplicity, we study almost monochromatic tau leptons from the decay of the virtual $\mathrm{Z}^{0} *, \mathrm{e}^{+} \mathrm{e}^{-} \rightarrow \mathrm{Z}^{0} * \rightarrow \tau^{+} \tau^{-}$at an $e^{ \pm}$beam energy of $125 \mathrm{GeV}$. This corresponds to a centre-of-mass energy of $250 \mathrm{GeV}$, modified by beamstrahlung and radiative corrections.

The momenta of the tau leptons from this process are larger than, for example, in $\mathrm{e}^{+} \mathrm{e}^{-} \rightarrow \mathrm{H}^{0} \mathrm{Z}^{0^{*}}, \mathrm{H}^{0} \rightarrow \tau^{+} \tau^{-}$at the same centre-of-mass energy. Therefore, our results correspond to Higgs boson production at higher ILC energies.

Only the task of the correct classification of the three $\tau^{+}$ decay modes mentioned above is considered in this paper. Backgrounds are not considered, and no attempt is made to extract the $\tau^{+}$spin state from the momenta of the reconstructed decay products.

The ILD detector has been optimized for the particle flow algorithm approach (PFA) [3,4] to event reconstruction. It combines very lightweight tracker systems with highly granular, "imaging", calorimeters. A complete description of ILD is given in $[5,6]$. In this study the ILD performance with a baseline design is simulated with the software framework MOKKA [7] with a parametric geometry description, based on the GEANT4 package [8]. The charged pion from $\tau^{+}$decay is reconstructed in the silicon vertex and tracker detectors and the time projection chamber. Photon clusters are reconstructed in the Si-W ECAL. We limit our analysis to the barrel part of ECAL, to avoid complications in the transition region

\footnotetext{
$\overline{1}$ The inclusion of charge-conjugate states is implied in this article.
} 
between the barrel and endcap. The hadronic calorimeter is not used.

The Si-W ECAL has a thickness of about 23 radiation lengths at normal incidence. The silicon sensors have a pixelization of $5 \times 5 \mathrm{~mm}^{2}$ and a thickness of $500 \mu \mathrm{m}$. Due to their high cost, and the large instrumented area, Si-W ECAL is one of the most expensive sub-detectors in ILD. Several studies of its cost optimization have been performed. In [6] it has been shown that a reduction of the number of ECAL layers from 30 to 26 degrades the jet energy resolution by less than $5 \%$ for jet energies in the range $45-250 \mathrm{GeV}$. In a more recent paper [9], it was demonstrated that a reduction of the ECAL radius by about $20 \%$ degrades the jet energy resolution by at most $8 \%$ in the same energy range, while the total cost of ILD could be lowered by a factor of 1.5 .

The current paper supplements the latter optimization study. In addition to the baseline ILD design with an ECAL inner radius of $1843 \mathrm{~mm}$, we present the results on tau decay mode reconstruction in smaller ILD models with inner ECAL radii of 1615 and $1450 \mathrm{~mm}$. These numbers have been chosen according to the size of the large industrially available silicon sensors in order to simplify the final ECAL mechanical design. The ECAL length is also reduced in order to leave the radius-to-length aspect ratio unchanged. All other detector parameters, such as the ECAL total and layer thicknesses, pixel size, gap between barrel and endcaps, etc., are unchanged.

The degradation of the jet energy and track momentum resolutions in a smaller ILD may be compensated by a higher magnetic field. Therefore, we have also simulated ILD performance with the magnetic field increased from the nominal 3.5 to $4 \mathrm{~T}$.

\section{Simulation and reconstruction}

We used a sample of $\mathrm{e}^{+} \mathrm{e}^{-} \rightarrow \mathrm{Z}^{0^{*}} \rightarrow \tau^{+} \tau^{-}(\gamma)$ decays at $250 \mathrm{GeV}$ produced for the ILD "Detailed Baseline Design" report included in the ILC "Technical Design Report" [6]. The tau decay is generated by the TAUOLA library [10]. Events with an initial state radiation are removed at a generator level, but events with final state radiation (FSR), $\mathrm{Z}^{0^{*}} \rightarrow \tau^{+} \tau^{-} \gamma$, are retained. Events with FSR in the tau decays, $\tau^{+} \rightarrow \pi^{+} \bar{v}_{\tau} \gamma, \rho^{+} \bar{v}_{\tau} \gamma, \mathrm{a}_{1}^{+} \bar{v}_{\tau} \gamma$, are also retained. The tau branching fractions of the three decays under study without FSR are listed in Table 1 . They cover $45 \%$ of all tau decay modes and about $70 \%$ of hadronic decays.

Each of the two $\tau$-leptons is reconstructed only inside a hemisphere defined by the axis pointing in the tau direction in the $\mathrm{Z}^{0^{*}}$ rest frame. Events in which a photon converts before the ECAL are removed by requiring that only one charged track is reconstructed in each hemisphere.
Table 1 Branching fractions of the three tau decays under study with one charged and up to two neutral pions in the final state [11]

\begin{tabular}{lc}
\hline Decay mode & Branching fraction [\%] \\
\hline$\pi^{+} \bar{v}_{\tau}$ & $10.83 \pm 0.06$ \\
$\rho^{+} \bar{v}_{\tau}\left(\rho^{+} \rightarrow \pi^{+} \pi^{0}\right)$ & $25.52 \pm 0.09$ \\
$\mathrm{a}_{1}^{+} \bar{v}_{\tau}\left(\mathrm{a}_{1}^{+} \rightarrow \pi^{+} \pi^{0} \pi^{0}\right)$ & $9.30 \pm 0.11$ \\
\hline
\end{tabular}

\subsection{The GARLIC clustering algorithm}

The Gamma Reconstruction at a Linear Collider Experiment (GARLIC) package [12,13], version 3.0.3, is used to find photon clusters. The calibration constants for converting the energy deposited in the silicon sensors to the full photon energy are determined using samples of $10 \mathrm{GeV}$ photons. This is done separately for the baseline and two smaller ILD ECAL models to take into account the possible variation of the overall weight of non-instrumented ECAL zones. The difference between the calibration constants of the three models is less than $1 \%$.

\subsection{Photon identification}

After finding clusters in the ECAL with the GARLIC package, their final identification as photon showers is performed using the following algorithm, which was specifically developed for the purposes of this analysis.

FSR photons are suppressed by requiring the photon energy to be greater than $0.5 \mathrm{GeV}$. This eliminates $70 \%$ of FSR photons and only $6 \%$ of photons from $\tau^{+} \rightarrow \rho^{+} \bar{v}_{\tau}$ and $\mathrm{a}_{1}^{+} \bar{v}_{\tau}$ decays.

To distinguish genuine photons from fake clusters originating, for example, from charged $\pi^{+}$hadronic showers, a boosted decision tree (BDT) with gradient boost is used with the following input variables:

- the distance from the cluster barycentre to the $\pi^{+}$track at the ECAL front surface;

- the cluster depth computed by the GARLIC algorithm;

- the cluster transverse fractal dimension defined as the logarithm of the number of hits in the cluster, $N_{1}$, divided by the number of hits when the pixels are grouped into larger pseudo-cells of $4 \times 4$ pixels, $N_{4}$ :

$F D_{4}=\log \left(N_{1} / N_{4}\right) / \log (4)$.

The fractal dimension, introduced in ref. [14], reflects the shower density which is higher in electromagnetic showers.

- the mean and standard deviation of the distribution of hit energies in the cluster; and 


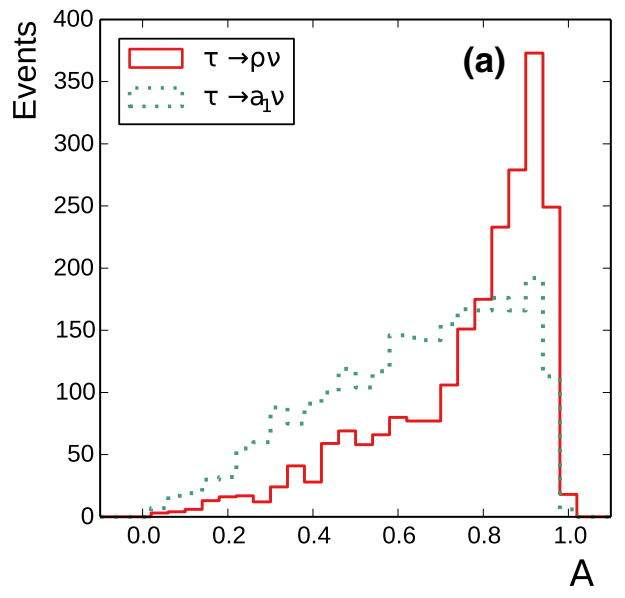

Fig. 1 The photon energy asymmetry $A=\left(E_{\gamma}^{\max }-\right.$ $\left.E_{\gamma}^{\min }\right) /\left(E_{\gamma}^{\max }+E_{\gamma}^{\min }\right)$ in the case of three (a) and two (b) reconstructed photons. Solid and dashed curves are for: a

- the fraction of cluster energy deposited between 5 and 10 radiation lengths after the starting point of the cluster.

$25 \%$ of all tau decays are used to train the BDT, which is then applied to the remaining $75 \%$ of tau decays. The photon clusters for which more than $50 \%$ of the hit energy is produced by the true Monte Carlo photon from tau decay are used as a signal for a BDT training. The remaining clusters in the same data sample serve as background.

The number of photons from $\tau^{+}$decay without FSR should be even. Sometimes, however, three photons are reconstructed. This can happen if one of the photons from $\tau^{+} \rightarrow \rho^{+} \bar{v}_{\tau}, \rho^{+} \rightarrow \pi^{+} \pi^{0}, \pi^{0} \rightarrow \gamma \gamma$ decays is reconstructed as two clusters, or if the decay is accompanied by an FSR photon. The other possibility is the merging of two photons into a single cluster in $\tau^{+} \rightarrow \mathrm{a}_{1}^{+} \bar{\nu}_{\tau}, \mathrm{a}_{1}^{+} \rightarrow \pi^{+} \pi^{0} \pi^{0}$ decay, or loss of one photon. In the first case of $\tau^{+} \rightarrow \rho^{+} \bar{\nu}_{\tau}$ decay, the photon with smallest energy is soft and the asymmetry between maximal and minimal energies of three photons, defined as

$A=\frac{E_{\gamma}^{\max }-E_{\gamma}^{\min }}{E_{\gamma}^{\max }+E_{\gamma}^{\min }}$

peaks at one. The distribution of $A$ for both $\tau^{+} \rightarrow \rho^{+} \bar{\nu}_{\tau}$ and $\mathrm{a}_{1}^{+} \bar{\nu}_{\tau}$ is shown in Fig. 1a. In this special case of three photons, if $A>0.8$, the energy of the least energetic photon cluster is added to the most energetic one and the cluster is removed from the following analysis.

As a cross-check, the same asymmetry $A$ is plotted in Fig. $1 \mathrm{~b}$ for the case of two reconstructed photons. It is dominated by $\tau^{+} \rightarrow \rho^{+} \bar{v}_{\tau}$ decays. Since $\pi^{0}$ has no spin, its decay products should have a flat asymmetry distribution, ranging from zero to one since the photon is massless. This

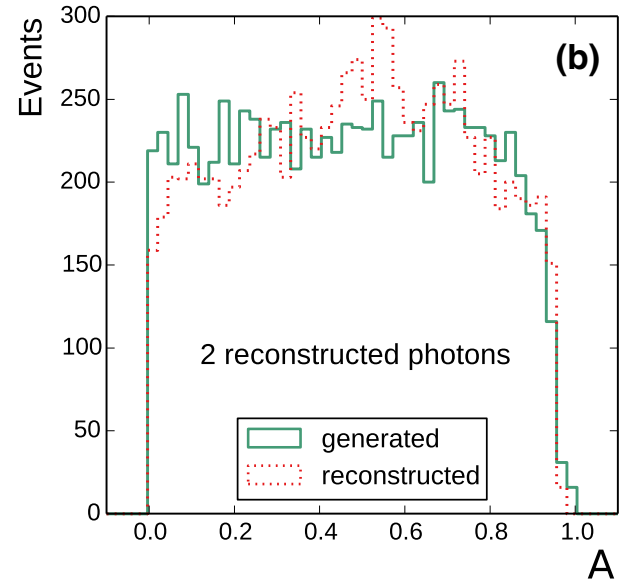

two decay modes $\tau^{+} \rightarrow \rho^{+} \bar{\nu}_{\tau}$ and $\tau^{+} \rightarrow \mathrm{a}_{1}^{+} \bar{\nu}_{\tau}$; and $\mathbf{b}$ for the generated (Monte Carlo truth) and measured energies, respectively

is indeed the case for the true Monte Carlo photon energies, save for effects of detector acceptance at one (the solid curve in Fig. 1b). The dashed curve, obtained after reconstruction, shows that the clustering algorithm tends to favor an asymmetric energy partition between two nearby photons.

\subsection{Classification of tau decay modes}

To distinguish the three considered $\tau^{+}$decay modes, a second BDT with gradient boost is used with the following input variables:

- the invariant mass of all reconstructed $\tau^{+}$decay products, $M_{\text {reco; }}$;

- the number of reconstructed photons;

- their total invariant mass;

- the energy of the photons; and

- their distance from the reconstructed track at the ECAL front surface.

For every decay mode a BDT is trained on $25 \%$ of statistics using this decay as a signal and the other two as a background. The three classifiers obtained in this way (one for each mode) are applied to the remaining $75 \%$ of events.

If $M_{\text {reco }}$ is larger than the tau lepton mass, the event is rejected, except if it is classified as $\pi^{+} \bar{\nu}_{\tau}$ with one reconstructed photon. In the latter case it is assumed that the mass beyond the kinematical limit is due to an external FSR photon, and the event is accepted.

A small fraction of events is accepted by two classifiers, mainly when the final state with three reconstructed photons is compatible with both $\rho^{+} \bar{\nu}_{\tau}$ and $\mathrm{a}_{1}^{+} \bar{\nu}_{\tau}$ decays. In such cases the final decision is made based on $M_{\text {reco }}: \rho^{+} \bar{\nu}_{\tau}$ is preferred over $\mathrm{a}_{1}^{+} \bar{v}_{\tau}$ if $M_{\text {reco }}<0.85 \mathrm{GeV}$, and vice versa. Events selected by other combinations of two classifiers $\left(\pi^{+} \bar{\nu}_{\tau}\right.$ and 
$\rho^{+} \bar{\nu}_{\tau}$ or $\pi^{+} \bar{\nu}_{\tau}$ and $\mathrm{a}_{1}^{+} \bar{\nu}_{\tau}$ ) are assigned to the final state with larger mass. No event passes all three classifiers.

\section{Results and conclusions}

Figure 2 summarizes the probability of correct reconstruction of the tau decay modes $\tau^{+} \rightarrow \pi^{+} \bar{\nu}_{\tau}, \rho^{+} \bar{v}_{\tau}$ and $\mathrm{a}_{1}^{+} \bar{\nu}_{\tau}$, in the central barrel region of the $\mathrm{Si}-\mathrm{W}$ ECAL, and in the absence of backgrounds. The results are presented for the ILD baseline design with an inner ECAL radius $R_{\mathrm{ECAL}}^{\text {inner }}=1843 \mathrm{~mm}$, and for two smaller ILD models with $R_{\mathrm{ECAL}}^{\text {inner }}=1615$ and $1450 \mathrm{~mm}$. The probability does not include the efficiency of charged $\pi^{+}$reconstruction, which is taken to be $100 \%$. The off-diagonal values correspond to the probabilities of incorrect reconstruction. For some events the decay mode could not be determined (either $M_{\text {reco }}$ is larger than the tau lepton mass or the event is not accepted by any classifier, as explained in Sect. 2.3), therefore the sums of the probabilities in columns and rows do not reach $100 \%$. The reconstruction of two tau leptons in $\mathrm{Z}^{0^{*}} \rightarrow \tau^{+} \tau^{-}(\gamma)$ events is performed independently, so that a failure to reconstruct one does not affect the other. The quoted uncertainties are purely statistical.

The correct reconstruction probability is close to $90 \%$ for $\rho^{+} \bar{\nu}_{\tau}$ and $\mathrm{a}_{1}^{+} \bar{v}_{\tau}$ and above $95 \%$ for $\pi^{+} \bar{\nu}_{\tau}$ decays. For the smallest ILD with $R_{\mathrm{ECAL}}^{\mathrm{inner}}=1450 \mathrm{~mm}$ the degradation of the probabilities compared to the baseline design is less than $2 \%$.

The slightly degraded jet and track momentum resolution of smaller ILD models can be compensated by a higher magnetic field. This increases the bending of charged tracks, and therefore the typical separation between charged hadrons pairs, and between charged hadrons and photons. We therefore also simulated the ILD performance with the magnetic

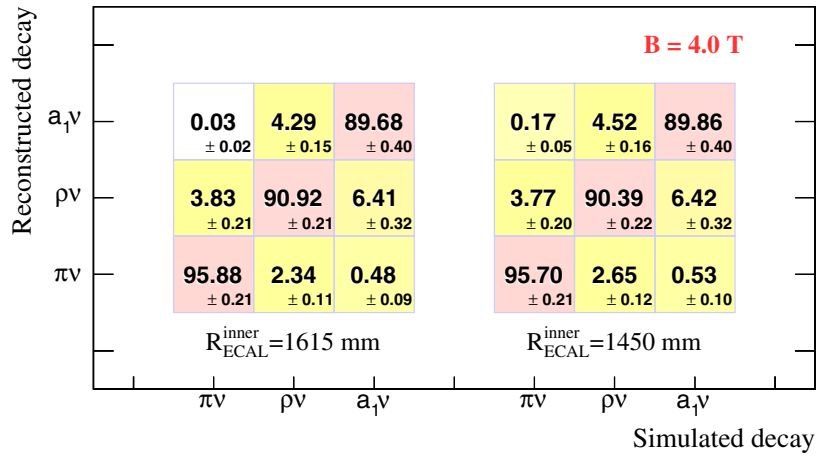

Fig. 3 The same as in Fig. 2, but for the magnetic field of $4 \mathrm{~T}$

field increased from the nominal 3.5 to $4 \mathrm{~T}$. The corresponding reconstruction probabilities are shown in Fig. 3. The improvement is marginal, less than $1 \%$. This demonstrates that the failure rate is dominated by photon reconstruction which is almost independent of the magnetic field.

The photon clusters from the decay of energetic $\pi^{0}$ s often overlap, which degrades the $\pi^{0}$ mass resolution. The effect is stronger for smaller ILD ECAL models, which tend to have less separation between photons. We study this effect using a sample with two reconstructed photons dominated by $\rho^{+} \bar{v}_{\tau}$ $\left(\rho^{+} \rightarrow \pi^{+} \pi^{0}\right)$ decays, and therefore typically containing a rather energetic $\pi^{0}$. Figure 4 a shows that the reconstructed invariant mass of the $\pi^{0}$ increases with its energy. This is explained by the fact that the GARLIC algorithm splits the overlapping showers geometrically into two disjoint groups of neighboring hits, neglecting the fact that some part of the cluster energy can propagate into the area of another cluster. This tends to overestimate the distance between the cluster barycenters and the opening angle between the photons. The distribution in Fig. $4 \mathrm{a}$ is fit to a parabolic function and the corresponding correction is applied to recover the nominal $\pi^{0}$ mass at all energies. The resulting mass resolution,

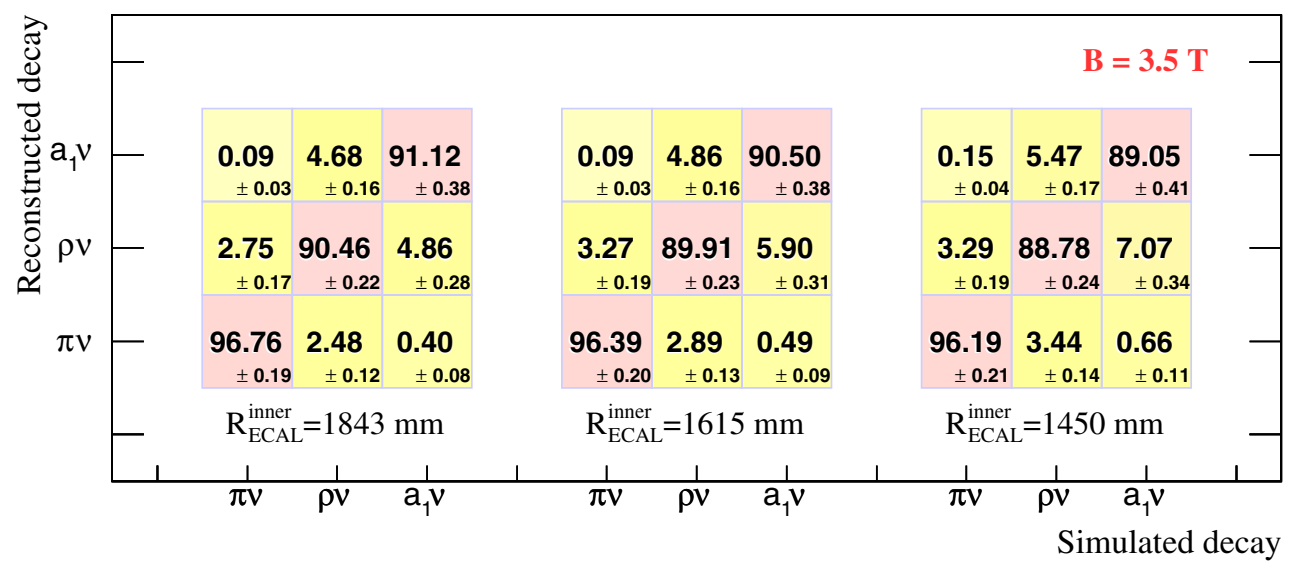

Fig. 2 The probabilities of correct and wrong reconstruction of the tau decay modes $\tau^{+} \rightarrow \pi^{+} \bar{\nu}_{\tau}, \rho^{+} \bar{\nu}_{\tau}$ and $\mathrm{a}_{1}^{+} \bar{\nu}_{\tau}$ in percent, obtained for models with $\mathrm{Si}-\mathrm{W}$ ECAL inner radius (from left to right) $R_{\mathrm{ECAL}}^{\mathrm{inner}}=1843$ (baseline), 1615 and $1450 \mathrm{~mm}$, and with the nominal magnetic field of 3.5 T. Only statistical uncertainties are shown 

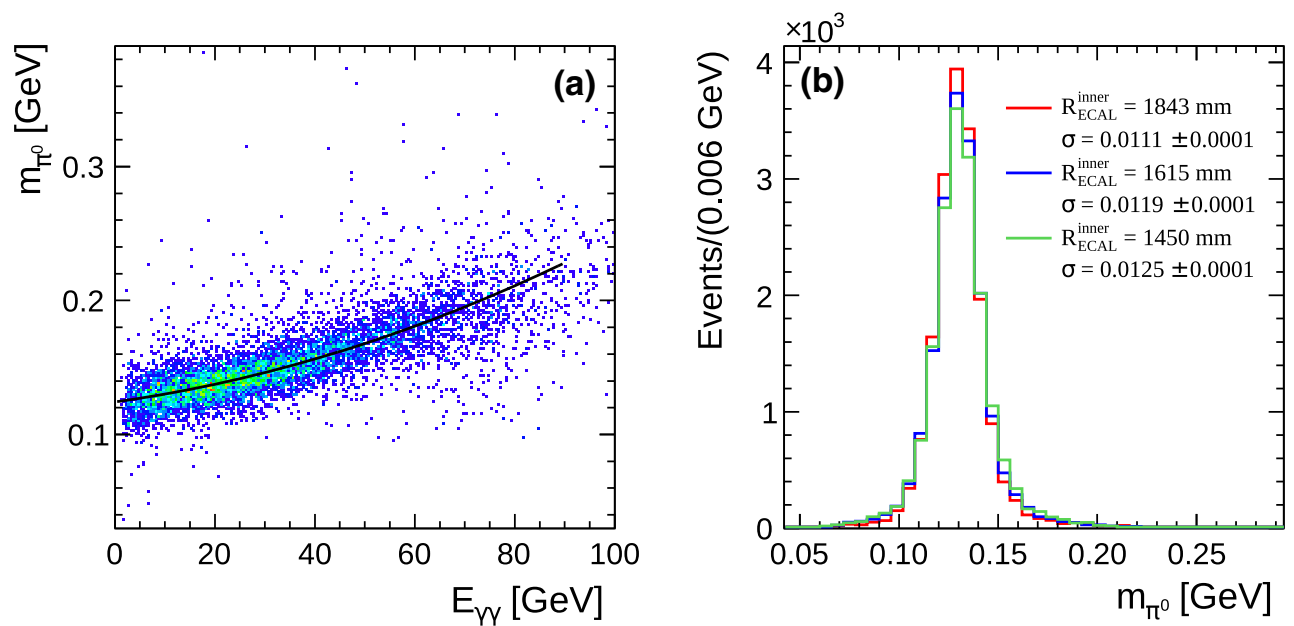

Fig. 4 a The dependence of the reconstructed mass of $\pi^{0}$ on its energy before the correction. The black curve shows the parabolic fit. $\mathbf{b}$ The corrected $\pi^{0}$ mass and the resolution for ILD with three different ECAL radii

shown in Fig. 4b, has a symmetric shape. The relative $\pi^{0}$ mass resolution is below $10 \%$ for all considered ILD models. The difference in mass resolution between the nominal and smallest ILD models is about $1 \%$.

A proper reconstruction of the tau decay modes together with a good momentum measurement of the decay products is mandatory to reconstruct the spin states of tau lepton in the measurement of the Higgs boson CP state in the decay $\mathrm{H}^{0} \rightarrow \tau^{+} \tau^{-}$. The high probability of the correct decay mode reconstruction demonstrated in this paper shows the full potential of the ILD, even with reduced size, for such a measurement.

Acknowledgments The authors would like to thank Dr. Daniel Jeans, University of Tokyo for the help with the GARLIC package used in this study. This work is funded by the Physics Department of École polytechnique, Palaiseau, France and the "Physique des deux Infinis et des Origines" (P2IO) program.

Open Access This article is distributed under the terms of the Creative Commons Attribution 4.0 International License (http://creativecomm ons.org/licenses/by/4.0/), which permits unrestricted use, distribution, and reproduction in any medium, provided you give appropriate credit to the original author(s) and the source, provide a link to the Creative Commons license, and indicate if changes were made.

Funded by SCOAP ${ }^{3}$.

\section{References}

1. A. Heister et al. (ALEPH collaboration), Eur. Phys. J. C 20, 401 (2001). arXiv:hep-ex/0104038

2. See, for example, the paper S. Berge, W. Bernreuther, H. Spiesberger, Phys. Lett. B 727 488, (2013). arXiv:1308.2674 [hep-ph] and many references therein
3. J.-C. Brient, H. Videau, in Proccedings of APS/DPF/DBP Summer Study on the Future of Particle Physics (Snowmass, Colorado, 2002). arXiv:hep-ex/0202004

4. M.A. Thomson, Nucl. Instrum. Methods A 611, 25 (2009). arXiv:0907.3577 [physics.ins-det]

5. The ILD Concept Group, "ILD Letter of Intent", DESY 2009-87, KEK 2009-6 (2010). arXiv:1006.3396 [hep-ex]

6. T. Behnke et al., The ILC Technical Design Report. Int. Linear Collid. 4 (2013). arXiv:1306.6329 [physics.ins-det]

7. P.M. de Freitas, H. Videau, Detector simulation with MOKKA/GEANT4: Present and future. in Proccedings of International Workshop on Linear Colliders (LCWS, Jeju Island, Korea, 2002), pp. 26-30

8. S. Agostinelli et al., Nucl. Instrum. Methods. A 506, 250 (2003). SLAC-PUB-9350, FERMILAB-PUB-03-339

9. T.H. Tran, in Proccedings of International Workshop on Future Linear Colliders (LCWS13, Tokyo, Japan). arXiv:1404.3173 [physics.ins-det]

10. S. Jadach, J.H. Kühn, Z. Was, Comput. Phys. Commun. 64, 275 (1990)

11. K.A. Olive et al., Chin. Phys. C 38, 090001 (2014)

12. M. Reinhard, J.-C. Brient, in Proccedings of International Linear Collider Workshop (LCWS08 and ILC08, Chicago, Illinois). arXiv:0902.3042 [hep-ex]

13. D. Jeans, J.-C. Brient, M. Reinhard, J. Instrum. 7, P06003 (2012). arXiv:1203.0774 [physics.ins-det]

14. M. Ruan, D. Jeans, V. Boudry, J.-C. Brient, H. Videau, Phys. Rev. Lett. 112, 012001 (2014). arXiv:1312.7662 [physics.ins-det] 